\title{
Colliding Ice Comets and the Reality of Energy
}

\author{
Peter J. Riggs a) \\ Department of Quantum Science, Australian National University, Canberra, ACT 0200, \\ Australia
}

(Published in: Physics Essays 23 (2010): 621-624)

\begin{abstract}
The reality of energy is occasionally questioned in the physics literature despite strong arguments and experimental evidence in favour of its existence. The Ice Comets Contention is a scenario where two comets made of ice collide and melt. The heat that melts the comets is assumed to 'flow' from one comet to the other but the direction of heat 'flow' is inferred to be observer dependent. This result is used to conclude that energy is not real. It is shown why this attempt fails.
\end{abstract}

Résumé: La réalité de l'énergie est parfois remise en cause dans les recherches en physique, et ce, malgré de forts arguments et des données expérimentales qui soutiennent son existence. L' «hypothèse des comètes de glace » est un scénario selon lequel deux comètes entrent en collision et fondent. On présuppose que la chaleur qui fait fondre les comètes passe d'une comète à l'autre mais le sens du courant de chaleur permet d'inférer qu'elle varie en fonction de l'observateur. Ce résultat est utilisé pour conclure que l'énergie n'est pas réelle. On montre pourquoi cette tentative échoue.

Key Words: Reality of energy; heat 'flow' direction; ice comets; Minkowski spacetime

a) peter.riggs@anu.edu.au 


\section{INTRODUCTION}

Whether energy is a real aspect of nature is both an appropriate and an important question to be addressed by fundamental physics for the following reasons: (i) all modern physical theories utilise energy as an indispensible concept; (ii) mathematical descriptions of physical systems are given in terms of energy processes and the conservation of energy; and (iii) explanations of cosmological significance (e.g. the 'Big Bang', the expansion of the universe, 'dark' energy, etc.) require energy to be a real attribute of physical systems. However, the question regarding the reality of energy has hardly been looked at in the physics literature whereas much discussion is to be found in science education journals. ${ }^{1,2,3,4,5}$ What mostly appears in physics articles is a plethora of material detailing formal aspects of energy such as spacetime energy conditions, spatial distributions of energy, conditions on field energy, thermodynamic properties of matter, and states of negative energy. ${ }^{6,7,8,9,10}$

A general definition of energy is not presently known. Instead, energy is defined in specific areas of physics, i.e. in mechanics, thermodynamics, quantum theory, etc. ${ }^{11}$ In the absence of a general definition, the well-known concept of energy and its characteristics (e.g. is conserved, able to be transferred and transformed), as set out in most university level physics textbooks, will be assumed. Physical implications for the reality of energy arising from (what shall be called) the Ice Comets Contention are explored in this paper, although no attempt is made to 'prove' the reality of energy.

\section{THE ICE COMETS CONTENTION}

The Ice Comets Contention is a deceptively simple scenario where two comets wholly made of ice are moving directly towards each other in outer space. They have no net electric charge, no rotational motion, and there are no external fields present in their neighbourhood (including gravitational). Consequently, the Contention does not involve 
potential energy. The comets collide, fuse, and then as a result of the heat produced, they melt. Prior to the collision, each comet constitutes an inertial frame of reference. Since each comet is at rest in own reference frame, it has zero kinetic energy relative to that frame. A principal claim made in this scenario is that an observer on either comet would determine that the heat that melts both comets 'flows' (i.e. heat is conducted) from the other comet, as the other comet appeared to have all the energy before collision. ${ }^{12}$ In other words, the inference made is that the direction of heat 'flow' after the collision of the comets is reference frame dependent. The conclusion then drawn is that the direction of heat 'flow' is not real (given the assumption that anything real should be independent of the frame of reference used in its description).

A further conclusion made using this scenario is that, since the direction of heat 'flow' is frame dependent and that kinetic energy is relative to one's particular frame of reference, energy cannot be a real physical quantity. This second conclusion has been employed to support the 'accountant's view' of energy which claims that energy is nothing more than a mathematical 'construct' used in scientific theories. ${ }^{13}$ In this view, the calculated (i.e. numerical) value of energy merely tallies up correctly if the appropriate sums are done, just like the book-keeping methods of financial accountants balance monetary credits and debits. ${ }^{14,15}$ The 'accountant's view' has some minority support. It will be shown that the above stated conclusions do not follow from the Ice Comets Contention.

A word about the terminology used in the Ice Comets Contention is in order. The term 'flow' suggests that heat is a fluid. Although the behaviour of heat has some characteristics similar to fluids, the suggestion is misleading. Energy may be transferred from one spatial region to another or from one object to another by well defined physical processes (i.e. conduction, convection, radiation) but the transfer of heat is not the flow of a fluid. ${ }^{16}$ However, as the Ice Comets Contention employs the term 'flow', 
we shall continue this usage but take it to refer to the process of conduction of heat through matter.

\section{NON-RELATIVISTIC CASE}

Let's consider the Ice Comets Contention from its original non-relativistic perspective by looking at the energy of the comets from two different frames of reference. It is assumed that both comets are of equal inertial mass $(m)$, where inertial mass is given by reference to Newton's Second Law. Let the motion of the comets be with respect to a suitable inertial frame of reference $\Sigma$ that is at rest relative to the 'fixed' stars. Let the comets be denoted (1) and (2) with comet (1) having a constant velocity $\mathbf{v}$ in the positive $\mathbf{x}$-direction of a Cartesian coordinate system at rest in frame $\Sigma$ and comet (2) with velocity $(-\mathbf{v})$. In this frame of reference, the comets will obviously have equal but opposite momenta and equal kinetic energies. The total momentum of the system consisting of both comets before collision in frame $\Sigma$ will be zero with total kinetic energy E:

$\mathrm{E}=1 /{ }_{2} m|\mathbf{v}|^{2}+1 /{ }_{2} m|-\mathbf{v}|^{2}=m|\mathbf{v}|^{2}$

The collision is purely inelastic as the comets form a single body on impact (which then melts). Conservation of total momentum requires that this single body is at rest in frame $\Sigma$ and all of the kinetic energy of the system is transformed into the heat Q that melts the cometary matter.

Now consider the situation from the rest frame of one of the comets, say comet (2).

Denote this reference frame as $\Sigma^{\prime}$. An observer on comet (2) would see comet (1) racing towards comet (2) with relative speed $2|\mathbf{v}|$. Therefore the total momentum of the system before collision in frame $\Sigma^{\prime}$ will be $2 m \mathbf{v}$ with the value of total kinetic energy being:

$E^{\prime}=1 / 2 m(2|\mathbf{v}|)^{2}+0=2 m|\mathbf{v}|^{2}$ 
All the kinetic energy of the system, according to the observer in frame $\Sigma^{\prime}$, resides with comet (1). (Of course, if we had instead considered an observer on comet (1), a similar perspective would apply in relation to comet (2).) Further, it is obvious that eq. (2) gives twice the value of total kinetic energy as does eq. (1). Conservation of total momentum in frame $\Sigma^{\prime}$ before collision requires that the single body formed (of mass $2 m$ ), which quickly melts, will be moving with speed $|\mathbf{v}|$ after collision. (Any observer on comet (2) prior to the collision would need to get off it in order to remain in frame $\Sigma^{\prime}$ but we need not consider such practicalities.) Half of the initial kinetic energy in frame $\Sigma^{\prime}$ before collision remains as kinetic energy and half appears as heat after collision.

If the direction of heat 'flow' is taken as not being real (on the basis that either comet could appear to have all the energy before collision) then this can support the additional claim that energy is not real either for if energy is real then it is to be expected that the heat 'flow' direction would be real too. This claim that energy is not real also draws on the result that the value of the total kinetic energy of the system before collision is not the same in the two frames of reference. The implication can then be made is that energy is a computational 'book-keeping device' (the 'accountant's view').

There are at least three significant points that arise from the Ice Comets Contention. First, zero energy with respect to a particular reference frame is not necessarily equivalent to an absence of energy. If we shift the zero level for our reckoning of a body's energy by simply changing our reference frame, it does not follow that the body's energy has changed, only our determination of it. A suitable analogy is a temperature scale, for zero degrees Celsius (say) is not equivalent to a lack of temperature. ${ }^{17}$ Therefore, taking the perspective from frame $\Sigma^{\prime}$ does not necessitate that comet (2) had zero energy. 
Second, once the comets have collided the heat produced is generated in the region where the two comets have coalesced. This is because the heat is caused by friction between the atoms that successively form the leading edges of the two comets during the collision process. Yet, at the instant of impact, both comets will still be frozen. Any heat produced will distribute to the colder parts of the cometary matter until thermal equilibrium is achieved. Therefore, the heat generated in the collision process must dissipate over a finite amount of time (albeit a very small time interval) from the conjoined region into both comets. The heat 'flow' occurs in two directions! Stated more accurately, after collision heat is conducted through the matter which constituted the comets before collision from the region where the two comets coalesced. It is not the case that heat 'flows' from one comet to the other. What's more, the heat will be seen to 'flow' in two directions in all frames of reference. Since this is not a reference frame dependent phenomenon, as claimed in the Ice Comets Contention, it cannot be used to conclude anything about the non-reality of the direction(s) of heat 'flow'.

Third, since it is now clear that any heat 'flow' occurs in two directions from the junction region into both comets, this does not show (by itself) which $\operatorname{comet}(\mathrm{s})$ possessed energy before collision. Nor does consideration of the heat 'flow' indicate (by itself) how much energy could be attributed to either comet. On the basis of the direction of heat 'flow' in this scenario, it cannot be concluded that energy is not a real physical quantity. Importantly, these points hold regardless of whether we consider a non-relativistic or a relativistic description (to be discussed below).

In respect to the kinetic energy being greater in frame $\Sigma^{\prime}$ than in the frame $\Sigma$, the following rationale is typically invoked. In frame $\Sigma^{\prime}$, the kinetic energy of the system before collision is twice that in frame $\Sigma$. After the collision in frame $\Sigma^{\prime}$, half of the initial kinetic energy is transformed into the heat Q that melts the cometary ice and half 
continues as the kinetic energy of the joined body (albeit melted). This allows the same amount of energy to be turned into heat in both reference frames (an important consideration as Q could, in principle, be measured). However, this is just an application of the 'accountant's view' of energy, for the tally of energy in frame $\Sigma^{\prime}$ ensures that an equal amount of energy is allocated to heat (in both frames) without regard to anything else.

The proper assessment of the reality of energy by consideration of its magnitudes in different frames of reference needs to be done within a context where relativistic effects on physical quantities may be fully taken into account. Therefore, nonrelativistic physics is inadequate to the task of showing if energy is physically real or not. $^{18}$

\section{RELATIVISTIC CASE}

Let's now examine the energy of the comets in the two given frames of reference from a relativistic perspective. Assume that the inertial rest masses of the comets are both equal to $m_{\mathrm{O}}$ (where inertial mass is given by reference to the relativistic version of Newton's Second Law). As in the non-relativistic case, let comets (1) and (2) be moving with respect to frame $\Sigma$ directly toward each other with opposite velocities $\mathbf{v}$ and $(-\mathbf{v})$. In this frame, the relativistic energy before collision of comet (1) is: $\mathrm{E}_{1}=m_{\mathrm{o}} \gamma \mathrm{c}^{2}$, with the Lorentz factor $\gamma=1 /\left(1-v^{2} / c^{2}\right)^{1 / 2}$, and $v=|\mathbf{v}|$. Likewise, for comet (2), its energy before collision is: $\mathrm{E}_{2}=m_{\mathrm{o}} \gamma \mathrm{c}^{2}$. The total relativistic energy for the system consisting of both comets before collision in frame $\Sigma$ is:

$\mathrm{E}=\mathrm{E}_{1}+\mathrm{E}_{2}=2 m_{\mathrm{o}} \gamma \mathrm{c}^{2}$

In frame $\Sigma^{\prime}$, if comet (1) appears to approach comet (2) with a relative velocity $\mathbf{u}$, then the addition of relativistic speeds provides the magnitude of $\mathbf{u}$ given by: 
$\mathrm{u}=2 \mathrm{v} /\left(1+\mathrm{v}^{2} / \mathrm{c}^{2}\right)$

It can be seen in frame $\Sigma^{\prime}$, that the relativistic energy of comet (1) is: $\mathrm{E}_{1}{ }^{\prime}=m_{\mathrm{o}} \gamma^{\prime} \mathrm{c}^{2}$, with $\gamma^{\prime}=1 /\left(1-u^{2} / c^{2}\right)^{1 / 2}$ and the energy of comet (2) is: $E_{2}^{\prime}=m_{0} c^{2}$ (the comet's rest energy, i.e. the energy in a reference frame where its momentum is zero). Then the total relativistic energy $\mathrm{E}^{\prime}$ for the system before collision in frame $\Sigma^{\prime}$ will be:

$\mathrm{E}^{\prime}=\mathrm{E}_{1}{ }^{\prime}+\mathrm{E}_{2}{ }^{\prime}=m_{\mathrm{o}} \gamma^{\prime} \mathrm{c}^{2}+m_{\mathrm{o}} \mathrm{c}^{2}=m_{\mathrm{o}} \mathrm{c}^{2}\left(\gamma^{\prime}+1\right)$

The relativistic energy of the system in frame $\Sigma$ after collision has the value $2 m_{\mathrm{O}} \mathrm{c}^{2}$. Then the magnitude of the change in energy $\Delta \mathrm{E}$ of the system in frame $\Sigma$ is:

$\Delta \mathrm{E}=2 m_{\mathrm{O}} \gamma \mathrm{c}^{2}-2 m_{\mathrm{O}} \mathrm{c}^{2}=2 m_{\mathrm{O}} \mathrm{c}^{2}(\gamma-1)$

which is, of course, equal to the sum of the relativistic kinetic energies of the comets before collision in frame $\Sigma$. The well-known expansion for $\gamma=\left[1 /\left(1-v^{2} / c^{2}\right)^{1 / 2}\right] \approx[1+$ $\left.1 / 2\left(\mathrm{v}^{2} / \mathrm{c}^{2}\right)\right]$ gives an approximation for eq. (6) as: $\Delta \mathrm{E} \approx m_{\mathrm{o}} \mathrm{v}^{2}$. If we identify the inertial rest mass $m_{\mathrm{O}}$ with the non-relativistic inertial mass $m$, then we get the same result for the amount of kinetic energy transformed into heat $\mathrm{Q}$ as in the non-relativistic case.

Although conservation of total relativistic momentum requires that the system's mass after collision increases by $\Delta m=\left(\Delta \mathrm{E} / \mathrm{c}^{2}\right),{ }^{19,20,21}$ discussion of this issue is not crucial here and would lead into a much larger topic which depends on how both inertial mass (as appears in Newton's Second Law) and amount of matter are defined. ${ }^{22}$ This is beyond the scope of the current article.

Equation (5) gives the relativistic energy before collision in frame $\Sigma^{\prime}: m_{\mathrm{o}} \mathrm{c}^{2}\left(\gamma^{\prime}+1\right)$. After collision in frame $\Sigma^{\prime}$, the conjoined cometary material is moving with velocity $\mathbf{v}$ 
and consequently has relativistic energy $2 m_{\mathrm{o}} \gamma \mathrm{c}^{2}$. The change in energy $\Delta \mathrm{E}^{\prime}$ of the system in frame $\Sigma^{\prime}$ is therefore:

$\Delta \mathrm{E}^{\prime}=m_{\mathrm{O}} \mathrm{c}^{2}\left(\gamma^{\prime}+1\right)-2 m_{\mathrm{o}} \gamma \mathrm{c}^{2}$

From the definition of $\gamma^{\prime}$ and using eq. (4), we find that:

$\left(\gamma^{\prime}+1\right)=2 c^{2} /\left(c^{2}-v^{2}\right)$

With the use of eq. (8), we can see that eq. (7) becomes: $\Delta \mathrm{E}^{\prime}=2 m_{\mathrm{o}} \gamma \mathrm{c}^{2}(\gamma-1)=\gamma(\Delta \mathrm{E})$, by eq. (6). The most likely situation for objects such as comets is where the velocity is very much less than the speed of light $(\mathrm{v}<<\mathrm{c})$, then $\gamma \approx 1$ and $\Delta \mathrm{E}^{\prime} \approx(\Delta \mathrm{E}) \approx m_{\mathrm{O}} \mathrm{v}^{2}$. This then gives the same value for the change in total energy in both of the reference frames $\Sigma$ and $\Sigma^{\prime}$, unlike the total energy changes in the non-relativistic case. The presence of rest energy terms in relativistic energy equations is indispensable in obtaining this result. $^{23}$

What about when velocities very close to the speed of light are involved? Although highly unlikely for comets, the situation of extreme relativistic velocities brings out very interesting aspects of the energy question for then the approximation $\Delta \mathrm{E}^{\prime} \approx(\Delta \mathrm{E})$ does not hold and instead we need the exact equality, $\Delta \mathrm{E}^{\prime}=\gamma(\Delta \mathrm{E})$. In other words, the change in total energy in each of the respective reference frames is directly related by the Lorentz factor $\gamma$. What the presence of the Lorentz factor indicates is that the proportionality between $\Delta \mathrm{E}$ and $\Delta \mathrm{E}^{\prime}$ is a consequence of the structure of Minkowski spacetime. ${ }^{24,25}$ This structure is also responsible for the existence of the more familiar relativistic effects (such as time dilation and length contraction). It presents a circumstance fundamentally different from the classical case, for velocities being purely additive in classical physics (i.e. consistent with Galilean transformations) can be taken 
to imply that kinetic energy is not physically real (as seen above). Importantly, Minkowski spacetime provides an arena for interactions in which physical attributes (such as energy) cannot be divorced from spacetime structure. ${ }^{26,27,28}$ This is not well appreciated but is essential to understanding the nature of energy.

Now, in respect to the reality of relativistic energy, it has been claimed that energy-momentum is the real physical quantity rather than relativistic energy as energymomentum has the same value in all inertial reference frames. ${ }^{29}$ This claim about the non-reality of relativistic energy may be answered as follows. The well-known relation for the total relativistic energy $\mathrm{E}$ of an object of inertial rest mass $m_{\mathrm{O}}$ is:

$E=\left[|\mathbf{p}|^{2} c^{2}+\left(m_{\mathrm{O}} c^{2}\right)^{2}\right]^{1 / 2}$

where the relativistic momentum $\mathbf{p}=m_{\mathrm{o}} \gamma \mathbf{v}$ (with the object's velocity $\mathbf{v}$ given relative to a suitable frame which is external to the object $), \gamma=1 /\left(1-v^{2} / c^{2}\right)^{1 / 2}$ and $v=|\mathbf{v}|$. The term $\left(m_{\mathrm{O}} \mathrm{c}^{2}\right)$ in eq. (9) is, of course, the object's rest energy. Rest energy is not only reference frame independent, it is the object's intrinsic energy content. ${ }^{30,31}$ The unequal values of total relativistic energy found in different frames of reference (with relative velocity very close to the speed of light) is therefore due to the different determinations of relativistic kinetic energy in each frame which appear through the presence of the momentum dependent term $\left(|\mathbf{p}|^{2} \mathbf{c}^{2}\right)$ in eq. (9). Clearly, it would be completely at odds with what we understand a relativistic frame of reference to be if the value of an object's relativistic kinetic energy did not alter from one reference frame to another. The relativistic kinetic energy $\mathrm{T}$ of the object is defined as: ${ }^{30}$

$\mathrm{T}=\mathrm{E}-m_{\mathrm{O}} \mathrm{c}^{2}=m_{\mathrm{O}} \mathrm{c}^{2}(\gamma-1)$

where $\mathrm{E}=m_{\mathrm{o}} \gamma \mathrm{c}^{2}$. The term $\left(|\mathbf{p}|^{2} \mathrm{c}^{2}\right)$ can be expressed explicitly as a function of T. From eqs (9) and (10), we get: 
$|\mathbf{p}|^{2} \mathrm{c}^{2}=\mathrm{E}^{2}-m_{\mathrm{o}}{ }^{2} \mathrm{c}^{4}=m_{\mathrm{o}}{ }^{2} \mathrm{c}^{4}\left(\gamma^{2}-1\right)=[(\gamma+1) /(\gamma-1)] \mathrm{T}^{2}$

The presence of the terms involving the Lorentz factor $\gamma$ in eq. (11) shows that the determination of E (as specified by eq. (9)) cannot be wholly separated from the structure of Minkowski spacetime. Given this, the claim that relativistic energy is not a physically real quantity has as much potency as claiming that say, size is not a real physical aspect of an object because it is spacetime intervals (rather than spatial intervals) that are Lorentz invariant.

When the role of spacetime structure in interactions and the other aspects of relativistic energy are taken into account then it becomes apparent that the 'accountant's view' that energy is merely a computational 'book-keeping device' is not supported from a relativistic perspective.

\section{CONCLUDING REMARKS}

A number of important aspects have been overlooked in the original formulation of the Ice Comets Contention. The conclusions drawn from this scenario regarding both the non-reality of the direction of heat 'flow' after the comets have collided and of energy itself do not follow. Considerations of relativistic energy show that the 'accountant's view' of energy is not supported in a relativistic context. The difficulties encountered in dealing with the energy of a physical system in different reference frames reflect more on how our theories of spacetime deal with objects involved in interactions rather than whether energy is a real physical quantity. The issues concerning the nature of energy are complex and involved. The resolution of questions arising from these issues is not assisted by drawing conclusions from inadequate or overly simplified scenarios.

\footnotetext{
${ }^{1}$ J.W. Warren, Eur. J. Sci. Educ. 4, 295-297 (1982).
} 
${ }^{2}$ C.H. Poon, Phys. Educ. 21, 307-316 (1986).

${ }^{3}$ J. Beynon, Phys. Educ. 29, 86-88 (1994).

${ }^{4}$ M. Bunge, Sci. \& Educ. 9, 457-461 (2000).

${ }^{5}$ M. Hecht, The Physics Teacher 41, 486-493 (2003).

${ }^{6}$ M. Visser, S. Kar and N. Dadhich, Phys. Rev. Lett. 90, 201102 (2003).

${ }^{7}$ A. Borde, L.H. Ford and T.A. Roman, Phys. Rev. D 65, 084002 (2002).

${ }^{8}$ L.H. Ford, Int. J. Mod. Phys. A 25, 2355 (2010).

${ }^{9}$ F. Strub and J.-P. Bédécarrats, Int. J. Thermodynamics 3, 35 (2000).

${ }^{10}$ E.W. Davis and H.E. Puthoff, AIP Conf. Proc 813, 1362 (2006).

${ }^{11}$ P.J. Riggs, Quantum Causality: Conceptual Issues in the Causal Theory of Quantum Mechanics (Springer, Dordrecht, 2009), chapter 4.

${ }^{12}$ M. Lange, An Introduction to the Philosophy of Physics: Locality, Fields, Energy, and Mass (Blackwell, Oxford, 2002), p.210.

${ }^{13}$ V.J. Stenger, The Comprehensible Universe (Prometheus, Amherst, 2006), p.159.

${ }^{14}$ I. Sefton, Proceedings of the 9th Science Teachers Workshop (Science Foundation for Physics, Sydney, 2002), p.2.

${ }^{15}$ M. Lange, An Introduction to the Philosophy of Physics: Locality, Fields, Energy, and Mass (Blackwell, Oxford, 2002), p.123.

${ }^{16}$ A.J. Chapman in R.G. Lerner and G.L.Trigg (eds), Encyclopedia of Physics (Wiley-VCH, Chichester, 2005), pp.982-984.

${ }^{17}$ M. Bunge, Sci. \& Educ. 9, (2000), p.459.

${ }^{18}$ M. Lange, An Introduction to the Philosophy of Physics: Locality, Fields, Energy, and Mass (Blackwell, Oxford, 2002), p.157.

${ }^{19}$ P.G. Bergmann, Introduction to the Theory of Relativity (Dover, New York, 1976), p.93.

${ }^{20}$ H. Goldstein, Classical Mechanics (Addison-Wesley, Reading, MA., 1980), p.308.

${ }^{21}$ H. Weyl, Space-Time-Matter (Dover, New York, 1952), pp.202-204.

${ }^{22}$ M. Jammer, Concepts of Mass in Contemporary Physics and Philosophy (Princeton University Press, Princeton, 2000), chapter 3.

${ }^{23}$ E.F. Taylor and J.A. Wheeler. Spacetime Physics (Freeman \& Co., San Francisco, 1966), p.117.

${ }^{24}$ R. Torretti, Relativity and Geometry (Pergamon, Oxford, 1983), pp.96-97. 
${ }^{25}$ N.A. Doughty. Lagrangian Interaction: An Introduction to Relativistic Symmetry in Electrodynamics and Gravitation (Addison-Wesley, Sydney. 1990), p.301.

${ }^{26}$ R. Torretti, Relativity and Geometry (Pergamon, Oxford, 1983), p.98.

${ }^{27}$ S.Y. Auyang, How Is Quantum Field Theory Possible? (Oxford University Press, New York, 1995), p.31.

${ }^{28}$ C. Ray, Space, Time and Philosophy (Routledge, London \& New York, 1991), p.138.

${ }^{29}$ M. Lange, An Introduction to the Philosophy of Physics: Locality, Fields, Energy, and Mass (Blackwell, Oxford, 2002), p.223.

${ }^{30}$ W. Rindler, Essential Relativity: Special, General, and Cosmological (Springer, Berlin, 1977), p.83.

${ }^{31}$ R. Angel, Relativity: The Theory and its Philosophy (Pergamon, Oxford, 1980), p.85. 